\title{
Review of: "Sex- and estrous-cycle dependent dorsal hippocampal phosphoproteomic changes induced by low-dose ketamine"
}

\author{
Carlos M. Contreras ${ }^{1}$
}

1 Universidad Veracruzana

Potential competing interests: The author(s) declared that no potential competing interests exist.

The aspect of sex differences in the manifestations of depression and response to antidepressants is of current interest. The theme is interesting by itself, and authors found interesting data that merits a profound reflection about the theme. There are many observations coming from clinic and lab research pointing out a clear sex difference in these aspects, not fully explained nowadays.

This is a complete, interesting study. In general, the manuscript is well written and clear. The hypothesis of work is well explained and supported by recent bibliography. The experimental design is cute, and procedures followed in complete agreement with hypothesis. Description of results is complete and clearly enriched illustrations. Discussion and conclusions are also clear.

In short, I have no more comments for this manuscript that merits high priority for publication. 